\title{
Application of Luenberger's Observer in RFPT-based Adaptive Control - A Case Study
}

\author{
Krisztián Kósi \\ Doctoral School of Applied Informatics \\ Óbuda University \\ Budapest, Hungary \\ Email:kosi.krisztian@phd.uni-obuda.hu
}

\author{
József K. Tar, Tamás Haidegger \\ Antal Bejczy Centre of iRobotics \& \\ Institute of Applied Mathematics, John von Neumann Faculty of Informatics \\ Óbuda University \\ Budapest, Hungary \\ Email: \{tar.jozsef@nik., haidegger@ \}uni-obuda.hu
}

\begin{abstract}
The traditional way of thinking in controller design prefers the use of the "state space representation" introduced by R. Kalman in the early sixties of the past century. This system description is in close relationship with linear or at least partly linear system in which the linear part can be used in forming a quadratic Lyapunov function in the stability proof. In the standard model of such systems it is assumed that the state of the system is not directly observable, only certain linear functions of the state variable are directly measurable. Since such approaches introduce certain feedback gains for the state variable, observers are needed that calculate the estimation of the state variable on the basis of directly measurable quantities. The Luenberger observers solve this task via introducing a differential equation for the estimated state.

In order to avoid the mathematical difficulties of Lyapunov's "direct method" the "Robust Fixed Point Transformations (RFPT)" were introduced in a novel adaptive technique that instead of the state space representation directly utilized the available approximate model of the system to estimate its "response function". In this approach it was assumed that the system's response is directly observable and an iterative sequence was generated by the use of "Banach's Fixed Point Theorem" that converged to an appropriate deformation of the rough initial model to obtain precise trajectory tracking.

In the present paper it is shown that the Luenberger observers and the RFPT-based mathod can be combined in a more conventional approach of the adaptive controllers that are designed on the basis of finding appropriate feedback gains. Illustrative simulation examples are presented to substantiate this statement.
\end{abstract}

\section{INTRODUCTION}

In his survey book V. Jurdjevic [1] so characterized the "classic approaches of linear control" that were prevailing before the early publications of Rudolf Kalman in the sixties of the past century (e.g. [2]):

"Control theory, originally developed to satisfy the design needs of servomechanisms, under the name of "automatic control theory", became recognized as a mathematical subject in 1960, with the publication of the early papers of $R$. Kalman. Kalman challenged the accepted approach to control theory of that period, limited to the use of Laplace transforms and frequency domain, by showing that the basic control problems could be studied effectively through the notion of the state of the system that evolves in time according to ordinary differential equations in which controls appear as parameters. Aside from drawing attention to the mathematical content of control problems, Kalman's work served as a catalyst for further growth of the subject. Liberated from the confines of the frequency domain and further inspired by the development of computers, automatic control theory became the subject matter of a new science called systems theory."

Proceeding along these "fixed rails" even Isidori's book on nonlinear control systems [3] starts with the investigation of the "Linear Time-Invariant (LTI)" systems of the form

$$
\begin{gathered}
\dot{x}=A x+B u, \\
y=C x
\end{gathered}
$$

in which $A$ is a constant real matrix of size $n \times n, B$ is also a constant real matrix of size $n \times m(0 \leq m \leq n)$, $u$ of size $m \times 1$ is the array of the control signals, and $C$ is a constant real matrix of size $k \times n(k \leq n)$, and $y$ is the directly measurable quantity (it is assumed that the components of $x$ are not immediately observable. The possession of the "system model" means that we have exact information on matrices $A, B$, and $C$. If these matrices have explicit time-dependence through the parameters of the system we have "Linear Parameter Varying (LPV)" systems. If these matrices also depend on time through certain components of the state variable $x$ the system is categorized as "Quasi-Linear Parameter-Varying ( $q L P V)$ ". This latter concept was found to be very fruitable since with the combination of the "Tensor Product (TP)" model and the "Higher Order Singular Value Decomposition (HOSVD)" it allowed the utilization of the results obtained for linear controllers via solving linear matrix inequalities (e.g. [4], [5]).

Returning to the classic LTI systems, the system is regarded as controllable is from each posible initial state $x_{i}$ each possible final state $x_{f}$ can be reached. Furthermore, for realizing a control the state $x$ must be observable in order make it possible to ealize the necessary feedback. In the case of LTI systems the 1st equation of (1) can be regarded as an inhomogeneous set of linear differential equations of constant coefficients the general solution of which can be obtained as the sum of the general solution of the homogeneous set plus a particular solution of the inhomogeneous equations. This latter can be obtained as 


$$
x(t)=\int_{t_{0}}^{t} \exp (\xi A) B u(t-\xi) d \xi .
$$

Taking into account that $\exp (\xi A) B=\sum_{s=0}^{\infty} \frac{\xi^{s} A^{s}}{s !} B$, according to the Cayley-Hamilton Theorem the linearly independent matrices can be only $\left\{B, A B, A^{2} B, \ldots, A^{n-1} B\right\}$, the LTI system is controllable if these matrices have full rank [6]. Otherwise there were sub-spaces in $x$ the propagation of which could not be influenced by the components of $u$. In similar manner, if $C$ is not quadratic and directly invertible the sequence of the observed quantity and its derivatives can be considered as $\left\{y, \dot{y}, \ldots, y^{(n-1)}\right\}$ that can be obtained by substituting of the 1 st equation of (1) into its 2 nd equation leading to the coefficients of $x$ as $\left\{C, C A, C A^{2}, \ldots, C A^{n-1}\right\}$ in the linear equation

$$
\left[\begin{array}{c}
y \\
\dot{y} \\
\ddot{y} \\
\vdots \\
y^{(n-1)}
\end{array}\right]=\left[\begin{array}{c}
C \\
C A \\
C A^{2} \\
\vdots \\
C A^{(n-1)}
\end{array}\right] x+\text { known terms. }
$$

Again, due to the Cayley-Hamilton theorem no higher derivatives must be investigated. If the matrix in (3) has a non-zero null space then certain subspaces in $\{x\}$ cannot be observed. Regarding stability, if the real part of each eigenvalue of $A$ is negative, the system is stable, otherwise it is unstable.

For the cases in which in (1) $C$ has full rank (or a similar matrix equation can be obtained), since $x$ is not known, the appropriate feedback can be realized through its estimate $\hat{x}$ and the following system of differential equations:

$$
\begin{gathered}
\dot{x}=A x+B u, \\
\dot{\hat{x}}=A \hat{x}+B u+L(y-C \hat{x})
\end{gathered}
$$

that corresponds to the application of a Luenberger observer with the gains given in matrix $L$. The aim of this observer is the realization of the $\hat{x}(t) \rightarrow x(t)$ convergence while the feedback in the control signal $u$ is realized by the use of $\hat{x}$ instead of the unknown $x$. For simultaneous design of the feedback coefficients in $u$ and the matrix $L$, depending on the particular properties of the system under control, various possibilities exist. In the next section a "school example" will be considered.

\section{The System Model And the Conventional DESIGN}

Our model is a nonlinear oscillator having the exact equations of motion as

$$
\ddot{q}=-k q-b \dot{q}-c \dot{q}^{2}+u
$$

in which $k>0$ is a spring stiffness constant, $b>0$ describes viscous friction, and $c>0$ corresponds to the coefficient of the drag force while moving in e.g. air or water. This latter term makes the system's dynamics nonlinear. For the controller design the linear approximation of (5) is applied as

$$
\ddot{q}=-k q-b \dot{q}+u
$$

for which a linear design is possible as follows. The formal state-space model can be constructed by the introduction of the state variable $x:=\left[x_{1}, x_{2}\right]^{T}=[q, \dot{q}]^{T}$ leading to the reformulation of the approximate model as

$$
\left[\begin{array}{c}
\dot{x}_{1} \\
\dot{x}_{2}
\end{array}\right]=\left[\begin{array}{cc}
0 & 1 \\
-k & -b
\end{array}\right]\left[\begin{array}{l}
x_{1} \\
x_{2}
\end{array}\right]+\left[\begin{array}{l}
0 \\
1
\end{array}\right] u
$$

that corresponds to (1) with $A=\left[\begin{array}{cc}0 & 1 \\ -k & -b\end{array}\right]$ and $B=$ $\left[\begin{array}{l}0 \\ 1\end{array}\right]$ and a one dimensional control signal $u \in \Re$. It is worthy of note that in the "Adaptive Inverse Dynamics" and the "Adaptive Slotine-Li Robot Controller" [7] a similar step is done with the aim of obtaining a Lyapunov equation that contains the appropriate matrix $A$.

If the task is to trace a nominal trajectory $x^{N}(t):=$ $\left[q^{N}(t), \dot{q}^{N}(t)\right]^{T}$, in the possession of the exact model and the state variable $x$ the following feedback rule could be introduced: $B u^{N}(t):=\dot{x}^{N}(t)-A x^{N}(t)$ :

$$
\left[\begin{array}{c}
0 \\
u^{N}
\end{array}\right]=\left[\begin{array}{c}
\dot{x}_{1}^{N} \\
\dot{x}_{2}^{N}
\end{array}\right]-\left[\begin{array}{cc}
0 & 1 \\
-k & -b
\end{array}\right]\left[\begin{array}{c}
x_{1}^{N} \\
x_{2}^{N}
\end{array}\right]
$$

leading to $u^{N}=k x_{1}^{N}+b x_{2}^{N}$ without any contradiction since $\dot{x}_{1}^{N}=x_{2}^{N}$. This term can be completed by an error-feedback as $B u(t)=B u^{N}(t)+B\left[K_{1}, K_{2}\right]\left(x^{N}(t)-x(t)\right)$ in which $K_{1}$ and $K_{2}$ are feedback gains in $K:=\left[K_{1}, K_{2}\right]$. In this manner the following equations of motion can be obtained:

$$
\begin{gathered}
\dot{x}=A x+B u^{N}+B K\left(x^{N}(t)-x(t)\right) \\
\dot{x}^{N}=A x^{N}+B u^{N} \Rightarrow \\
\frac{d}{d t}\left(x^{N}-x\right)=(A-B K)\left(x^{N}-x\right)
\end{gathered}
$$

with $A-B K=\left[\begin{array}{cc}0 & 1 \\ -k-K_{1} & -b-K_{2}\end{array}\right]$. In this simple case the $x \rightarrow x^{N}$ convergence can be guaranteed by the simple method of Pole Placement i.e. by prescribing the spectrum of $A-B K$ in the following manner:

$$
\begin{gathered}
\operatorname{det}(A-B K-\lambda I)=\left(\lambda-\lambda_{1}\right)^{2} \Rightarrow \\
\lambda^{2}+\lambda\left(b+K_{2}\right)+k+K_{1}=\lambda^{2}-2 \lambda_{1} \lambda+\lambda_{1}^{2}
\end{gathered}
$$

yielding $K_{1}=\lambda_{1}^{2}-k$ and $K_{2}=-b-2 \lambda_{1}$ with $\Re \ni \lambda_{1}<0$.

If we assume that only $x_{1}$ can directly be observed the $y=[1,0]\left[x_{1}, x_{2}\right]^{T}=x_{1}$ rule is valid with $C=[1,0]$ in (1), i.e. we need one derivation as

$$
\left[\begin{array}{c}
y \\
\dot{y}
\end{array}\right]=\left[\begin{array}{c}
{[1,0]\left[x_{1}, x_{2}\right]^{T}} \\
{[1,0]\left[\dot{x}_{1}, \dot{x}_{2}\right]^{T}}
\end{array}\right]=\left[\begin{array}{c}
x_{1} \\
\dot{x}_{1}
\end{array}\right]=\left[\begin{array}{c}
x_{1} \\
x_{2}
\end{array}\right]
$$

therefore our complete set of equations will be

$$
\begin{gathered}
\dot{x}=A x+B u^{N}+B K\left(x^{N}-\hat{x}\right), \\
\dot{\hat{x}}=A \hat{x}+B u^{N}+B K\left(x^{N}-\hat{x}\right)+L(x-\hat{x})
\end{gathered}
$$


since the feedback is realized instead of $x$ by $\hat{x}$. For the state estimation error $e:=x-\hat{x}$ this yields

$$
\frac{d}{d t} e=A e-L e=(A-L) e .
$$

For the convergence $\hat{x} \rightarrow x$ the spectrum of $A-L$ must be properly set. Here $L$ denotes the feedback gain of the Luenberger Observer [8].

If $L$ is only a scalar then this spectrum is determined by the characteristic polynomial's roots that is by the solutions of the equation

$$
\operatorname{det}\left[\begin{array}{cc}
-L-\mu & 1 \\
-k & -b-L-\mu
\end{array}\right]=0
$$

yielding $\mu^{2}+(b+2 L) \mu+L(b+l)=0$, therefore $\mu_{1,2}=$ $\frac{-b-2 L \pm \sqrt{(b+2 L)^{2}-4 L(b+L)}}{2}$, that is $\mu_{1}=-L$ and $\mu_{2}=-b-L$ are negative values if $L>0$, therefore the $\hat{x} \rightarrow x$ convergence is granted for $L>0$. In the sequel the operation of this control designed for the linear approximation of the model is investigated for the nonlinear system. It is expected that for small coefficient $c$ it can work well but after transcending a limit for $c$ the controller may become unstable. Before providing simulation results the adaptive completion of this controller by the use of the RFPT transformations.

\section{THE RFPT-BASED DESIGN}

The RFPT-based method is an iterative technique designed for digital controllers of fixed cycle time. The iteration happens through the consecutive control cycles that for "Single InputSingle Output (SISO)" systems works as follows: The design is initiated by determining a kinematically prescribed desired system response $r_{n}^{D e s}$ that - in our case- will be $f:=\ddot{q}$. The control signal in cycle $n$ i.e. $u_{n}$ will be computed from that of cycle $n-1$ as $u_{n-1}$, and from the realized response observed in the previous cycle $f\left(u_{n-1}\right)$ as

$$
\begin{gathered}
u_{n}:=G\left(u_{n-1}, f\left(u_{n-1}\right), r_{n}^{\text {Des }}\right):= \\
\left(u_{n-1}+K_{c}\right) \times \\
\left(1+B_{c} \sigma\left(A_{c}\left[f\left(u_{n-1}\right)-r_{n}^{\text {Des }}\right]\right)\right)-K_{c}
\end{gathered}
$$

in which $\sigma(x) \in(-1,+1)$ is a monotone increasing smooth sigmoid function with the further properties as $\sigma(0)=0$, $\left.\frac{d \sigma(x)}{d x}\right|_{x=0}=1, B_{c}= \pm 1$, while $K_{c}$ and $A_{c}$ are adaptive control parameters [9]. The Response Function $f$ normally also depends on the actual state of the system, on the parameters of the approximate and the actual system models as well as on the external disturbances.

Regarding the convergence of the iteration Banach's Fixed Point Theorem [10] can be applied as follows: if the derivative of $G$ according to $u_{n}$ in its absolute value is smaller than 1 this function becomes contractive in the vicinity of the fixed point and it converges to this fixed point. The convergence can be achieved by properly setting the three adaptive control parameters $K_{c}, B_{c}$, and $A_{c}$. For improving the convergence properties of this novel methods complementary tuning techniques were recently suggested as e.g. [11], [12], [13].

\section{The Combination of the Traditional And the RFPT-BASED APPROACHES}

In the present context no complementary parameter tuning was necessary. In the "role" of the desired response $\ddot{q}$ that is the response of the approximate linear model was placed. The control signals were so deformed that the response of the nonlinear system well approximated that of the linear system. The nominal trajectory to be tracked was a third order spline function of time. The parameters were set as follows: $k=$ $100, b=10$ for the model, $\lambda_{1}=-12, L=24, K_{c}=10^{6}$, $B_{c}=-1, A_{c}=10^{-6}$.

Figure 1 demonstrates the operation of the Luenberger observer in the non-adaptive control for the exactly linear case in which $c=0$. It well corresponds to the theoretical expectations. Tis non-adaptive controller worked for $c=3$ (Fig. 2) but for higher values of $c$ it was found to be divergent (this corresponds to the limits of the linear design).
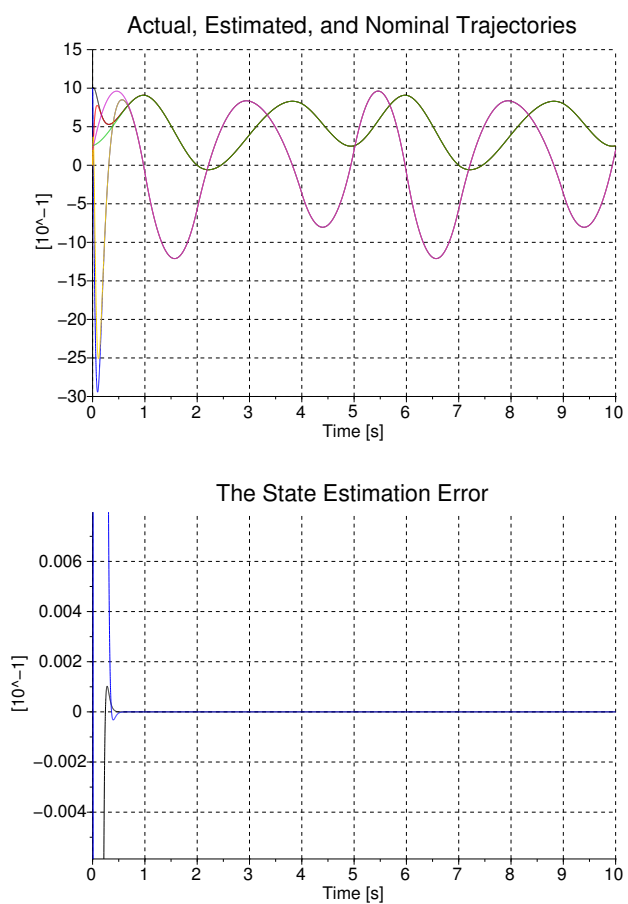

Fig. 1. The operation of the non-adaptive Luenberger observer for the exactly linear system model: trajectory tracking (top: $x_{1}$ : black, $x_{2}$ : blue, $\hat{x}_{1}$ : red, $\hat{x}_{2}$ ocher, $x_{1}^{N}$ : green, $x_{2}^{N}$ : purple lines) and the state estimation error (bottom) versus time in $[s]$ units

The adaptive counterpart of Fig. 2 is Fig. 3. The precision of the state estimation as well as that of the trajectory tracking were evidently improved.

The adaptive version was able to realize the control till $c=6$ (Fig. 4). The differences between the adaptive and nonadaptive control signals for $c=3$ and $c=6$ are given in Fig. 5. In Fig. 6 the control signals are described. It is worthy of note that the relative differences are small. However, if the fact is taken into account that the integral of the control signal is accumulated in time the significance of this little difference can well be understood. 

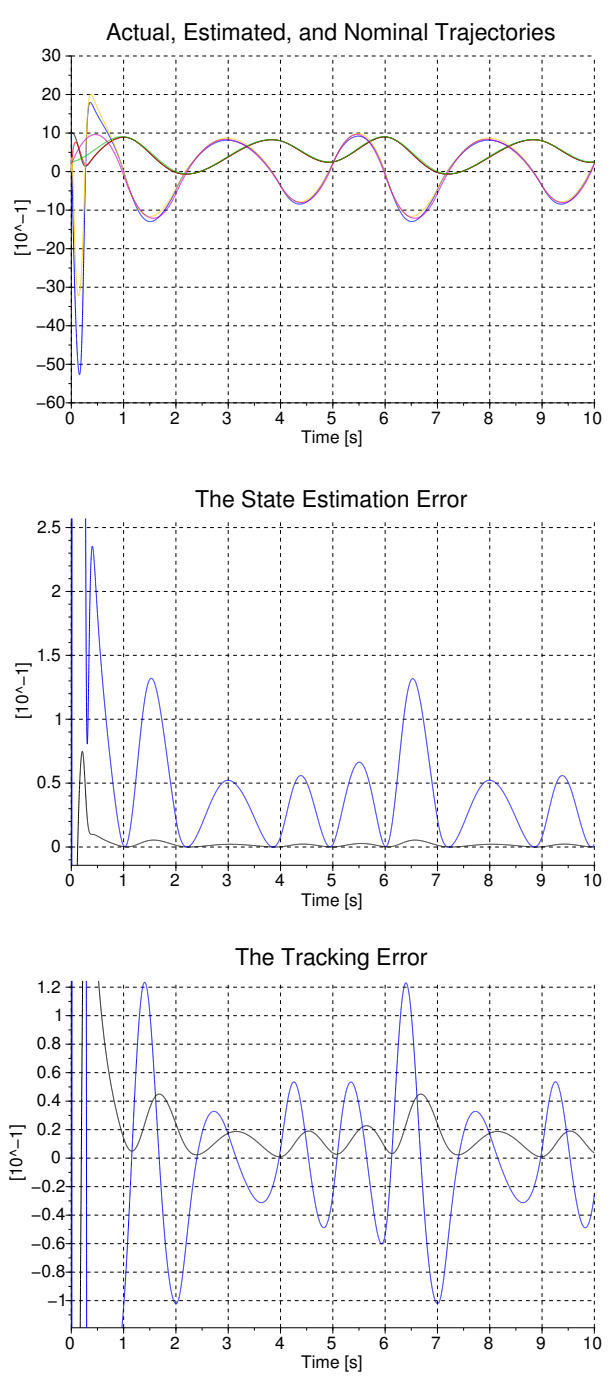

Fig. 2. The operation of the non-adaptive Luenberger observer for the modeling inaccuracy belonging to $c=3$ : trajectory tracking (top: $x_{1}$ : black, $x_{2}$ : blue, $\hat{x}_{1}$ : red, $\hat{x}_{2}:$ ocher, $x_{1}^{N}$ : green, $x_{2}^{N}:$ purple lines), the state estimation error (middle), and tracking error (bottom) [black line for $x_{1}$, and blue line for $\left.x_{2}\right]$ versus time in $[s]$ units

\section{Conclusions}

In this paper, via a simple case study, it was shown that the classical Luenberger observer and the RFPT-based adaptive controller needing immediately observed system response can be combined in order to serve the information-need of the RFPT-based controller. The here considered example was a nonlinear oscillator. Its linear approximation was used designing the feedback gains via pole placement and setting the gain of the Luenberger observer.

However, it must be stressed that the RFPT-based method can be applied independently of the "standard form" of (1) by directly using the approximate dynamic model of the system to be controlled. In this case the desired response can be directly designed without planning simple feedback gains as it is done in the case of real LTI systems.

Regarding further research it seems to be expedient to
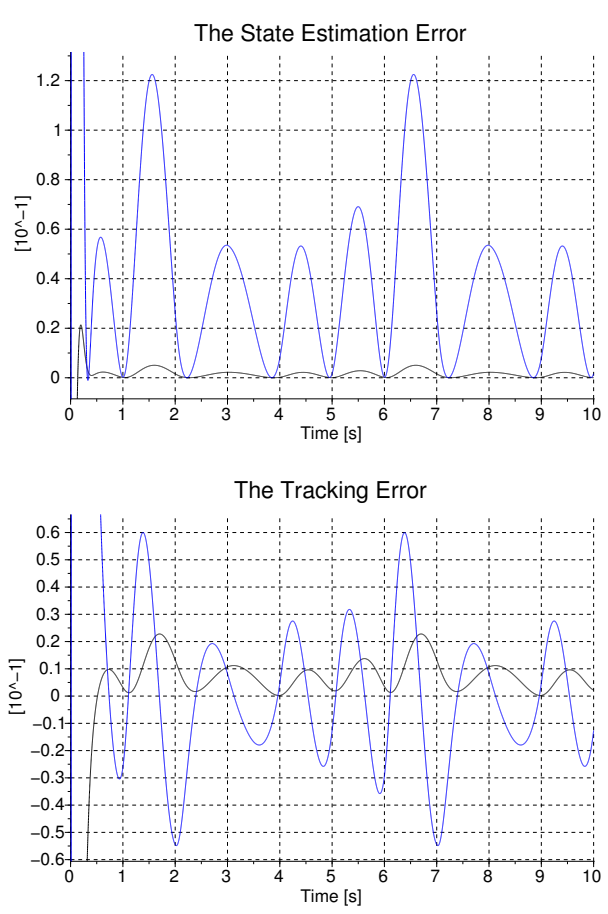

Fig. 3. The operation of the adaptive Luenberger observer for the modeling inaccuracy belonging to $c=3$ : the state estimation error (top), and tracking error (bottom) [black line for $x_{1}$, and blue line for $x_{2}$ ] versus time in $[s]$ units
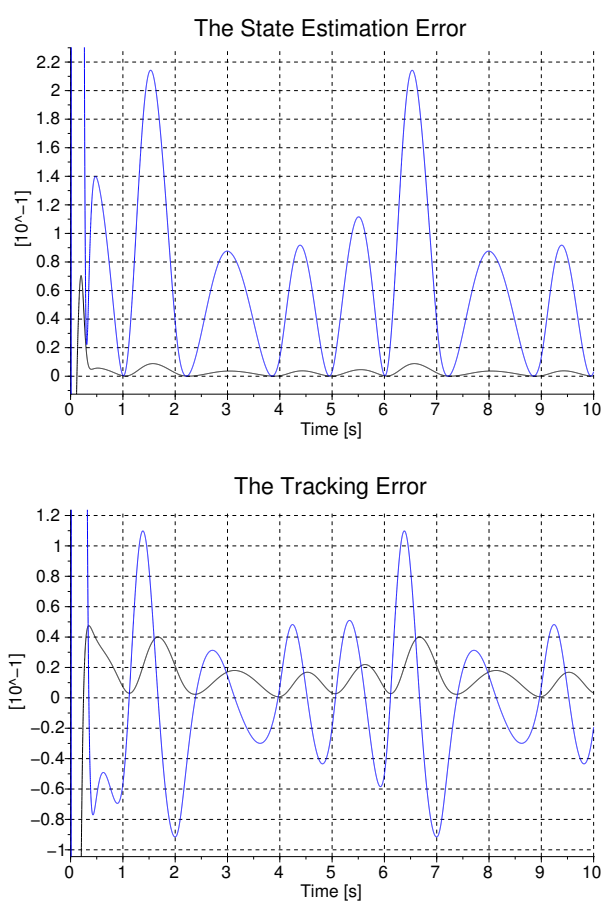

Fig. 4. The operation of the adaptive Luenberger observer for the modeling inaccuracy belonging to $c=6$ : the state estimation error (top), and tracking error (bottom) [black line for $x_{1}$, and blue line for $x_{2}$ ] versus time in $[s]$ units 

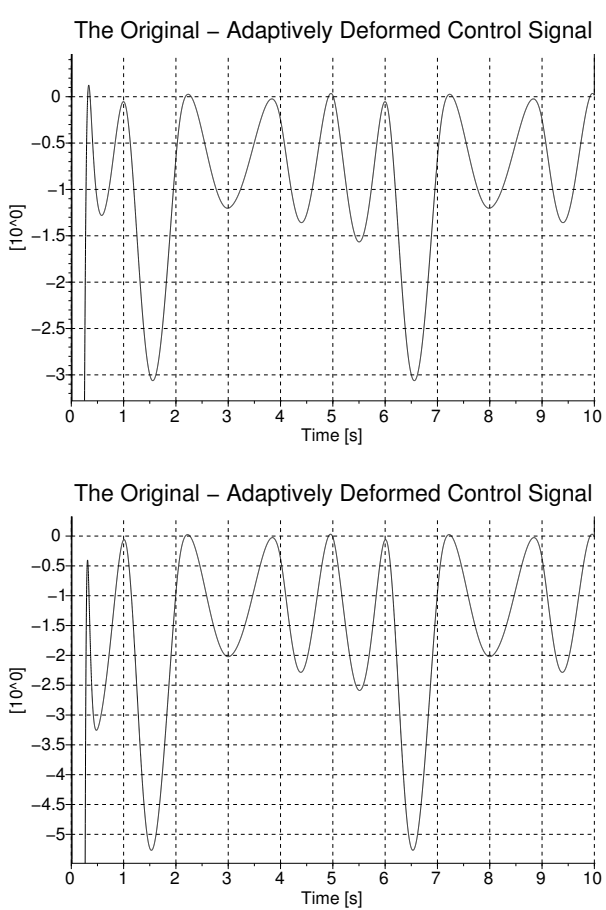

Fig. 5. The difference between the non-adaptive (black line) and adaptive (blue line) control signals for $c=3$ (top), and $c=6$ (bottom) versus time in $[s]$ units
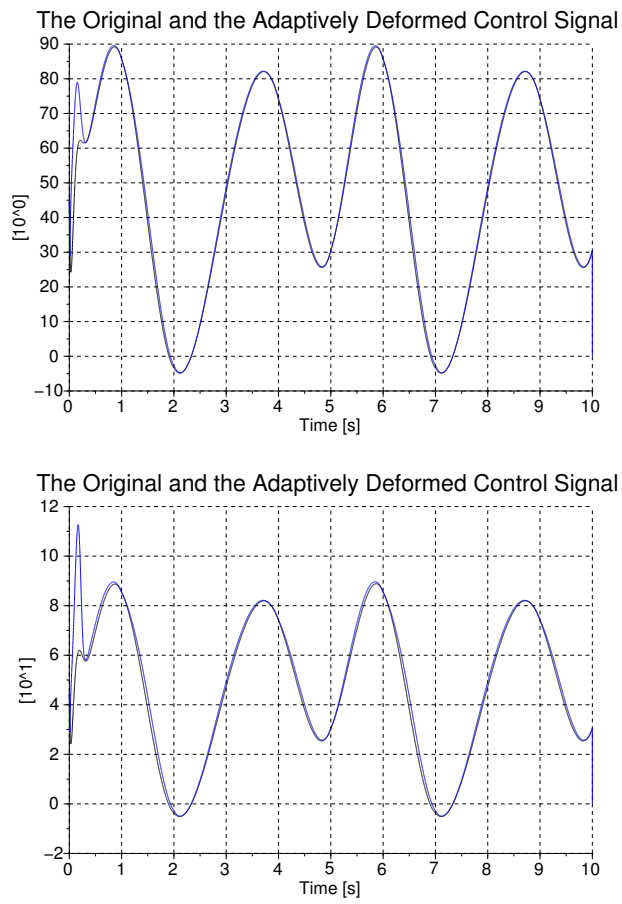

Fig. 6. The non-adaptive (black line) and adaptive (blue line) control signals for $c=3$ (top), and $c=6$ (bottom) versus time in $[s]$ units

make similar investigations for Kalman Filters and/or variable structure sliding mode filters for various nonlinear paradigms.

\section{ACKNOWLEDGMENT}

The authors thankfully acknowledge the grant provided by the Project TÁMOP-4.2.2.A-11/1/KONV-2012-0012: Basic research for the development of hybrid and electric vehicles - The Project is supported by the Hungarian Government and co-financed by the European Social Fund. We also express our thanks for the financial support by the Óbuda University Research Fund.

\section{REFERENCES}

[1] V. Jurdjevic. Geometric Control Theory. Cambridge University Press, 1997.

[2] R.E. Kalman. A new approach to linear filtering and prediction problems. Transactions of the ASME - Journal of Basic Engineering, 82 (Series D):35-45, 1960.

[3] A. Isidori. Nonlinear Control Systems (Third Edition). Springer-Verlag London Limited, 1995.

[4] Z. Petres, B. Reskó, and P. Baranyi. TP model transformation based control of the TORA system. Production Systems and Information Engineering, 2:159-175, 2004.

[5] P. Baranyi, Z. Petres, P. Várlaki, and P. Michelberger. Observer and control law design to the TORA system via TPDC framework. WSEAS Transactions on Systems, 1(5):156-163, 2006.

[6] S. Boyd. Introduction to Linear Dynamical Systems, Lecture Notes for EE263. Stanford University, 2007.

[7] Jean-Jacques E. Slotine and W. Li. Applied Nonlinear Control. Prentice Hall International, Inc., Englewood Cliffs, New Jersey, 1991.

[8] D.G. Luenberger. Introduction to Dynamic Systems: Theory, Models, and Applications. ohn Wiley \& Sons, New York, 1979.

[9] J.K. Tar, J.F. Bitó, L. Nádai, and J.A. Tenreiro Machado. Robust Fixed Point Transformations in adaptive control using local basin of attraction. Acta Polytechnica Hungarica, 6(1):21-37, 2009.

[10] S. Banach. Sur les opérations dans les ensembles abstraits et leur application aux équations intégrales (about the operations in the abstract sets and their application to integral equations). Fund. Math., 3:133-181, 1922.

[11] J.K. Tar. Towards replacing Lyapunov's 'direct' method in adaptive control of nonlinear systems (invited plenary lecture). In: Proc. of the 2010 Mathematical Methods in Engineering International Symposium (MME 2010), October 21-24, 2010, Coimbra, Portugal, 2010.

[12] J.K. Tar, L. Nádai, I.J. Rudas, and T.A. Várkonyi. RFPTbased adaptive control stabilized by fuzzy parameter tuning. 9th European Workshop on Advanced Control and Diagnosis (ACD 2011), Budapest, Hungary, pages $1-8,2011$.

[13] K. Kósi, J.K. Tar, and I.J. Rudas. Improvement of the stability of RFPT-based adaptive controllers by observing "precursor oscillations". In the Proc. of the 9th IEEE International Conference on Computational Cybernetics, 8-10 July 2013, Tihany, Hungary, pages 267-272, 2013. 